\title{
Behavioral Dyscontrol Following Acquired Brain Injury: Effectiveness of Post-hospital Neurobehavioral Intensive Programs
}

\author{
Gordon J. Horn ${ }^{1 *}$ \& Frank D. Lewis ${ }^{2}$ \\ 'Florida State University, College of Medicine, USA \\ ${ }^{2}$ Medical College of Georgia at Augusta University, USA
}

\section{Article Info}

\section{Article Notes}

Received: October 18, 2016

Accepted: November 22, 2016

\section{*Correspondence:}

Dr. Gordon Horn, Ph.D., NeuroRestorative National Clinical Outcomes, Florida State University, College of Medicine, USA, Telephone: (727) 647-0697;

Email: gordon.horn@neurorestorative.com

(c) 2016 Horn GJ. This article is distributed under the terms of the Creative Commons Attribution 4.0 International License

\section{Keywords:}

Acquired Brain Injury

Neurobehavioral

Outcomes

MPAI-4

Post-hospital rehabilitation

\begin{abstract}
The purpose of this research was to evaluate the effectiveness of post-hospital neurobehavioral intensive (NBI) programs for treating acquired brain injury survivors with significant symptoms of behavioral dyscontrol and to identify variables that predict functional outcome. Subjects were 219 adults with acquired brain injury (predominately traumatic brain injury, $81 \%$ ) exhibiting moderate to severe irritability, agitation, and/or aggression (includes verbal or physical) that were discharged from six NBI programs across five states. Prior to treatment, all participants demonstrated neurobehavioral impairment preventing the individuals from living in the community. All participants were assessed using the Mayo-Portland Adaptability Inventory - 4 at admission and discharge from program. A Repeated Measures MANOVA revealed significant improvement on the three MPAI-4 subscales at time of discharge. With control for participant age, a hierarchical multiple regression analysis revealed three significant MPAI-4 predictors of outcome: initiation, impaired awareness, and fund of information. Findings demonstrated that significant functional improvement can be realized with extremely chronic behaviorally intensive brain injured adults. Treatment effects may be enhanced by early intervention focused on appropriate response initiation/ inhibition, self-awareness of behavior on others, and information integration to facilitate appropriate response formation.
\end{abstract}

The percentage of brain injured patients discharged from acute hospital inpatient rehabilitation programs that exhibit persistent agitated behaviors is estimated to range between $11 \%$ and $34 \%^{1,2}$. Persons with significant behavioral dyscontrol after brain injury require intensive neurobehavioral rehabilitation from a skilled clinical team, because the most severe kinds of behaviors can result in potential danger to self and/or others. Behavioral dyscontrol includes: poor impulse control, explosive outbursts, verbal and physical aggression, poor planning and judgment, and/or limited or poor self-awareness ${ }^{3}$. As an example, self-awareness is the impact on others through behavior control and realistic appraisal through perspective taking. This adaptive skill is a hallmark impairment of neurobehavioral dysfunction ${ }^{4}$.

In a review, Nicholl and his colleagues ${ }^{5}$ emphasized that programs focusing only on cognitive and physical deficits after brain injury are unlikely to achieve positive outcomes for patients exhibiting these symptoms. Specialized post-hospital residential neurobehavioral intensive (NBI) programs teach patients how to use skills in context, using community outings and productive activities, rather than traditional therapies only. The short-term goal is to stabilize behavior with repeated learning trials so that patients can move to less restrictive, functionally-based settings ${ }^{3}$. The long-term goal is for patients to achieve socially appropriate behavior and selfmanaging skills so they can return home or live in the community with minimal to no supervision. The literature suggests that 
patients in NBI programs tend to be injured longer than the typical neurorehabilitation patients, with the injury onset to program admission interval often averaging greater than five years ${ }^{6}$. As time elapses without appropriate care, symptoms may worsen as survivors learn maladaptive ways of coping. Improved access to NBI programs would likely reduce: 1 ) the long-term burden of care (e.g., reduced need for supervision), 2) the risk to patient and caregiver, and 3) overall disability with more favorable outcomes. In order to achieve improved access, the effectiveness of these programs for treating the challenging symptoms of behavioral dyscontrol following brain injury, must be demonstrated.

Therefore, the present study addressed two questions: 1) Is post-hospital NBI programming effective in reducing disability resulting from acquired brain injury at the moderate to severe levels?, and 2) What are the variables that predict functional outcome?

\section{Methods}

\section{Subjects}

The study group was comprised of 219 adults with acquired brain injury who were discharged from six programs across five states that were designed to treat brain injured individuals exhibiting impulsivity along with verbal and/or physically aggressive behavior. Eightytwo percent of the sample was male. The average age was 37.3 years (range $=17-77$ years, $S D=13.2$ ). The average length of stay in the program was 12.4 months (range = 1-72 months, SD $=14$ months). A small number of cases $(\mathrm{N}=7)$ were in the program for 1 month due to loss of funding. Further analysis revealed no significant change from admission to discharge scores for this subgroup. The average chronicity (e.g., onset of injury to program admission) for the study group was 83.9 months (range = 1-529 months, SD = 116 months). Diagnoses included: TBI (81\%), cerebrovascular accident (3\%), anoxia (7\%), tumor (2\%), and medical/disease (7\%). The specialized treatment programs consisted of 7 days per week of neurobehavioral programming (i.e., use of behavior modification techniques in group and individual activities in the facilities and communities), 7 days per week of life skills application, and 5 days per week of neurorehabilitation intervention including occupational and speech therapies addressing functional deficits with cognitive and communication skills. Weekly psychological counseling was also utilized to promote prosocial behaviors.

Ninety-nine percent of the sample were rated as experiencing moderate to severe limitations in functioning on at least one of five key areas of the Mayo Portland Adaptability Inventory - 4 (MPAI-4). The five key areas that may characterize TBI survivors with behavioral dyscontrol include: irritability with verbal and/or physical aggression,

\begin{tabular}{|l|c|c|c|}
\hline MPAI-4 function & $\begin{array}{c}\text { Mild } \\
\text { Limitation }\end{array}$ & $\begin{array}{c}\text { Moderate } \\
\text { Limitation }\end{array}$ & $\begin{array}{c}\text { Severe } \\
\text { Limitation }\end{array}$ \\
\hline Irritability-Agitation & $43 \%$ & $35 \%$ & $22 \%$ \\
\hline Impaired Awareness & $21 \%$ & $28 \%$ & $51 \%$ \\
\hline Novel Problem Solving & $24 \%$ & $35 \%$ & $41 \%$ \\
\hline Impaired Initiation & $37 \%$ & $32 \%$ & $31 \%$ \\
\hline Family Relationship Impairment & $23 \%$ & $32 \%$ & $45 \%$ \\
\hline
\end{tabular}

Table 1: Percent of subjects with mild, moderate and severe limitations in MPAI-4 functions associated with behavioral dyscontrol.

impaired awareness of deficits, impaired novel problem solving, impaired initiation (appropriately engaging or inhibiting behavior), and disrupted family relationships. Table 1 presents the frequency of limitation in each of these areas by level of impairment.

\section{Treatment Setting}

Each of the six programs were designed exclusively to meet the care needs of NBI brain injured patients. Programs included a medical director (typically a Physiatrist with Psychiatric and Neuropsychological consultation) with a treatment team consisting of nursing, behavior specialists (i.e., staff trained specifically in behavior modification interventions), physical therapy (if indicated), occupational therapy, speech therapy, cognitive rehabilitation specialist, case management, and facility-based life skills teachers. Specifically, the behavioral specialists worked across disciplines to promote generalization of prosocial behaviors and mitigate negative behaviors with repeated learning trials for successful skills mastery.

Treatment facilities were designed to maximize safety and allow space for reducing stimulation and social complexity simultaneously. Large open areas created a better line of sight for nonintrusive supervision. Since aggression and agitation is significant in this population, these facilities employed applied behavior analysis to identify triggers that elicit aggression and to identify consequences maintaining aggression specifically. Once identified, these contingencies were modified to eliminate or substantially reduce aggressive behavior. Staff was trained in crisis prevention intervention and de-escalation techniques. Physicians prescribed and oversaw mood stabilizing medication, and counselors and behavior specialists instructed patients to replace maladaptive behaviors with prosocial ones.

\section{Measure}

Participant functioning was assessed using the MayoPortland Adaptability Inventory-4 (MPAI-4; Malec \& Lezak, 2008) at the time of admission and time of discharge from the treatment facilities involved in the study. Specifically, the MPAI-4 consists of 29 items rated from 0 to 4 on a 5-point scale, where 0 represents no limitations and 4 represents a severe problem interfering with activity more than $75 \%$ 
of the time. Raw scores on the 29 items are converted to T-scores within three subscales: Ability Index, Adjustment Index, and Participation Index. Each index has an average impairment T-score of 50 and a standard deviation of 10 points. The T-score interpretation is inverted so that higher scores reflect greater disability. The MPAI-4 and its three subscales (Ability, Adjustment, and Participation Indices) offer well developed and documented psychometric properties. Pearson Reliability studies for the MPAI-4 range from 0.78 to $0.88^{6}$. This level of reliability was achieved through MPAI-4 scoring by team consensus. The MPAI4 provides a comprehensive evaluation of the cognitive, physical, and behavioral sequelae following neurological injury. Additionally, the Participation Index provides a measure of the final common outcome aim - societal participation.

\section{Procedure}

Participants were evaluated upon admission by each program's multidisciplinary treatment team. Once individual discipline assessments were completed, each participant was then evaluated using the MPAI-4 within 30 days of admission by treatment team consensus. Discharge MPAI-4s were completed in a similar fashion (e.g., treatment team consensus) within the final days of the participant's stay. The assessment intervals were conducted in a manner consistent with the MPAI-4 guidelines within the administration manual based on the original reliability and validity studies ${ }^{7}$. The results of the evaluations were then compiled into a national database.

\section{Analysis}

A repeated measure multivariate analysis of variance (RM MANOVA) was provided to evaluate change scores with Abilities, Adjustment, and Participation Indices from admission to discharge. Efficacy of programming was determined by using this statistical approach.

Hierarchical regression analysis was conducted to determine the MPAI-4 variables that best predicted functional outcome at discharge. The participation T-score was used as the dependent variable in each of the regression analyses because it provides a measure of independent functioning. In cognitive neuroscience, there are five primary areas of cognitive functions with executive functions falling at the highest and most complex level. The primary reason executive functions are the highest level is because this system requires functioning and interaction of the other systems to produce cognitive, behavioral, communication and physical functioning to meet daily challenges ${ }^{8,9}$. The success of the interaction is based on the success of this system operating efficiently and effectively. Measuring the various primary and secondary functions of the frontal system is possible through the use of portions of the MPAI-4. The Abilities Index includes attention/concentration, fund of information, memory and novel problem solving. The Adjustment Index includes neurobehavioral components identified as IrritabilityAnger-Aggression, Impaired Social Interaction, and Impaired Awareness. The Participation Index measures the functional generalization of skills and Initiation, the ability to engage and inhibit behavior appropriately. As such, these variables were considered in the regression analyses as predictors of outcome. Other predictor variables included were length of stay, chronicity, and age.

\section{Results}

1. Effectiveness. Program effectiveness was evaluated by examining differences in MPAI-4 T-scores from admission to discharge. A Repeated Measures MANOVA revealed a significant main effect for pre-post testing, Pillai's Trace $=0.35, \mathrm{~F}=117.85, \mathrm{df}=(1,218), \mathrm{p}=.0005$, partial eta ${ }^{2}=.35$. Follow-up paired sample T-tests showed that for each subscale, participants made significant improvement in function from admission to discharge. Mean admission and discharge scores were: Abilities 52.2 vs 47.5, t(218)=9.2 $\mathrm{p}<.001$, Adjustment 58.8 vs 53.4, $\mathrm{t}(218)=9.7, \mathrm{p}<.001$, and Participation 55.8 vs $51.1, \mathrm{t}(218)=9.2, \mathrm{p}<.001$. This improvement in function is noteworthy given the subjects chronicity was approximately seven years.

2. Predictive Findings. Hierarchical multiple regression was conducted to determine the demographic and MPAI4 variables at admission that best predict functional outcome at discharge as measured by the Participation T-score. The following 6 MPAI-4 measures of frontallobe system functioning were entered into the regression equation: initiation, impaired awareness, novel problem solving, attention/concentration, fund of information, and memory. The demographic variables entered were age, length of stay, and chronicity interval. Prior to the analysis, tests for multicollinearity were conducted and revealed a very low association among predictor variables with Variable Inflation Factor (VIF) values of less than 2 for each comparison. With criterion for multicollinearity satisfied $^{10}$, a four stage hierarchical multiple regression analysis was performed. Age, length of stay, and chronicity interval were entered into the first hierarchical block. Together these variables predicted .068 of the variance of Participation T-scores at discharge (adjusted $\mathrm{R}^{2}=.054$, $\mathrm{F}(3,198)=4.82$, $\mathrm{p}<.005)$. Admission initiation scores were entered into the second block increasing the $\mathrm{R}^{2}$ to .281 (adjusted $\mathrm{R}^{2}=.267, \mathrm{~F}(1,197)=58.46 \mathrm{p}<.0005$ ). Inclusion of impaired awareness scores in the third block improved prediction by $7 \%\left(\mathrm{R}^{2}=.355\right.$, adjusted $\mathrm{R}^{2}=$ $.338, \mathrm{~F}(1,196)=22.26, \mathrm{p}<.0005)$. Finally, the fourth block consisted of four MPAI-4 cognitive variables: fund of information, attention/concentration, novel problem solving, and memory. These variables added an additional $4 \%$ to the 


\begin{tabular}{|l|l|l|l|}
\hline Block number and predictor & Final beta & R-square added & $\begin{array}{l}\text { Cumulative } \\
\text { R-square }\end{array}$ \\
\hline 1.Age & $0.250^{* * *}$ & & \\
\hline $\begin{array}{l}\text { LOS } \\
\text { Chronicity }\end{array}$ & 0.012 & $0.068^{* *}$ & $0.068^{* *}$ \\
\hline 2. Initiation & -0.043 & & \\
\hline 3. Impaired awareness & $0.275^{* * *}$ & $0.213^{* * *}$ & $0.281^{* * *}$ \\
\hline $\begin{array}{l}\text { 4. Fund of Information } \\
\text { Attention/Concentration }\end{array}$ & $0.196^{* *}$ & $0.073^{* * *}$ & $0.355^{* * *}$ \\
\hline $\begin{array}{l}\text { Novel Problem Solving } \\
\text { Memory }\end{array}$ & 0.087 & & \\
\hline
\end{tabular}

$* \mathrm{p}<.05 ; * * \mathrm{p}<.01 ; * * * \mathrm{p}<.001$

Table 2: Prediction of participation T-score at discharge for behavior intensive participants.

model increasing the $\mathrm{R}^{2}$ to its final level of .40, (adjusted $\left.\mathrm{R}^{2}=.369, \mathrm{~F}(4,192)=3.4, \mathrm{p}<.01\right)$. Table 2 displays the beta weights, predictive contributions and cumulative $\mathrm{R}^{2}$ for the model. An examination of the significant Beta weights with all predictors entered reveal that after controlling for age, initiation, impaired awareness, and fund of information made the greatest unique contributions to variance in Participation T-scores at discharge.

\section{Discussion}

The purpose of this research was to evaluate the effectiveness of post-hospital NBI rehabilitation and to identify those variables most important for achieving functional independence. A very positive finding of this study was that on average, participants realized meaningful reduction in disability from admission to discharge, thereby improving the capacity to function in the community. This is particularly noteworthy given the average chronicity of nearly 7 years. Nonetheless, participants demonstrated significant improvement on the MPAI-4 Abilities, Adjustment, and Participation T-scores from admission to discharge (mean LOS = 12.4 months). These findings offer a definitive answer to the first question posed in this study: post-hospital neurobehavioral intensive rehabilitation is effective in reducing functional disability following moderate to severe brain injury, even with chronically injured individuals with moderate to severe behavioral disorders.

The second purpose of this study was to identify those variables that have the greatest impact on functional outcome. Consistent with previous literature ${ }^{11}$, age was a significant predictor of outcome in this study, accounting for $7 \%$ of the variance in participation $\mathrm{T}$-scores at discharge specifically. Participants under the age of 50 had better functional outcomes than those over the age of $50, t(217)=2.8, p<.01$. After controlling for age, admission MPAI-4 variables initiation, impaired awareness, and fund of information added an additional $32 \%$ to the prediction, with initiation alone accounting for $21 \%$ of the variance in participation T-scores. At a functional level, the crux of the behaviourally intensive group is the inability to initiate and inhibit responses effectively (Initiation item), a limited understanding of their behavioral impact on interactions with others (Impaired Awareness item), and an inability to learn from past behavior based on a fund of knowledge encased in experience (Fund of Information item). The combined effect of all three variables creates the greatest challenge when away from a structured milieu. Most residential programs are highly structured but application of skills can be limited due to behavior risks. As such, treatment programs may consider emphasizing Initiation, Self-Awareness, and Information Integration for new response formation as a way to help participants manage more effectively and independently in less structured home and community settings.

\section{Conclusions}

The results of the study demonstrated evidence that improvement can be achieved with behaviorally intensive brain injured adults even with an extensive length of time since injury. The primary MPAI-4 predictors of positive outcome were Initiation, Impaired Awareness, and Fund of Information. The results of this research may provide a systematic method to formulate focused intervention strategies. These strategies may further enhance recovery with treatment modeling, and improve cost efficiency, for chronic brain injured survivors exhibiting significant behavior disorders.

\section{Limitations}

As is typical with applied clinical research, a nontreatment control group would not be possible to establish a comparison of outcomes. Also, the current research intent was on demonstrating the variables that are characteristic with neurobehavioral intensive subjects that have significant impact on program outcome. Subsequent research would then focus on long-term durability of postdischarge outcomes and societal participation.

\section{Acknowledgement}

This research was possible due to the financial support of NeuroRestorative.

\section{Funding of Information}

The research was funded by the employer of both authors. The research was completed as part of the national research program within NeuroRestorative.

\section{References}

1. Johnston M. Outcomes of community re-entry programmes for brain injury survivors Part 2 Further investigations. Brain Injury. 1991; 5(2):155-168.

2. Rao V, Rosenberg P, Bertrand M, et al. Aggression after traumatic brain injury Prevalence and correlates. Journal of Neuropsychiatry Clinical Neuroscience. 2009; 21(4): 420-429.

3. Horn G, Lewis F. A model of care for neurologic rehabilitation. Journal of Nurse Life Care Planning. 2014; 14(3): 681-692. 
4. Bivona U, Riccio A, Ciurli P, et al. Journal of Head Trauma Rehabilitation. 2014; 29(2):157-171.

5. Nicholl J, LaFrane C. Neuropsychiatric sequelae of traumatic brain injury. Seminars in Neurology. 2009; 29(3): 247-255.

6. Lewis FD, Horn GJ. Traumatic Brain Injury Analysis of functiona deficits and post- hospital rehabilitation outcomes. Journal of Special Operations Medicine. 2013; 13 (3): 56-61.

7. Malec JF, Lezak MD. The Mayo-Portland Adaptability Inventory (MPAI4) for adults children and adolescents. 2008; 1-84.
8. McDonald B, Flashman L, Saykin A. Executive dysfunction following traumatic brain injury Neural substrates and treatment strategies. NeuroRehabilitation. 2002; 17(4): 333-344.

9. Godefroy O. Frontal syndrome and disorders of executive functions. Journal of Neurology. 2003; 250 (1): 1-6.

10. Coakes S. SPSS: analysis without anguish: version12.0 for Windows. Singapore. John Wiley \& Sons Australia Ltd. 2005.

11. Marquez de la Plata C, Hart T, Hammond F, et.al. Impact of age on long-term recovery from traumatic brain injury. Archives of Physical Medicine and Rehabilitation. 2008; 89(5): 896-903. 